\title{
COMPUTER SIMULATION OF DIRECT OVERWRITE SCHEME IN THE EXCHANGE-COUPLED BILAYER FOR MAGNETO-OPTICAL MEMORY
}

\author{
M. HASEGAWA, K. MOROGA, M. OKADA*, O. OKADA* and Y. HIDAKA** \\ NEC Scientific Information System Development Co., Ltd., Takatsu-ku, Kawasaki 213, Japan \\ *Functional Devices Research Lab., NEC Corporation, Miyamae-ku, Kawasaki 216, Japan \\ **Hiroshima-Denki Institute of Technology, Nakano 6-20-1, Aki-ku, Hiroshima 793-03, Japan
}

\begin{abstract}
Computer simulation based on the Landau-Lifshitz-Gilbert equation, was developed and applied to analysing the reverse magnetic domain generation or its erasure in the light modulation overwrite medium, an exchange-coupled amorphous $\mathrm{TbFe} /$ GdTbFeCo bilayer film. It was found that the exchange coupling between the reference and the memory layers plays an essential role in replicating the domain from the reference to the memory layer. The dynamics of the boundary wall, which plays an important role in the erasure, was discussed, based on the correlation between the boundary wall width in the memory layer, $H_{B}$ and the temperature.

KEYWORDS: COMPUTER SIMULATION, OVERWRITE, EXCHANGE-COUPLED, LANDAU-LIFSHITZ-GILBERT EQUATION, LIGHT INTENSITY MODULATION, DOMAIN GENERATION, ALTERNATIVE DIRECTION IMPLICIT
\end{abstract}

\section{INTRODUCTION}

For overwrite in a magneto-optical (MO) disk system, a magnetic field modulation recording[1] and a light intensity modulation recording $[2]$ are representative techniques. Thermal recording is generally completed by two processes; (1)a reverse domain generation and(2) domain growth. In the conventional simulation, the latter process was mainly studied, provided the appropriate assumption was made for the former[3]. Final state of the written domain, however, is strongly influenced by the dynamic domain nucleation process, as well as the domain growth process. In order to simulate the domain growth, we developed a simulation program using the LandauLifshitz-Gilbert (LLG) equation, which treats the magnetization precession accurately through giving only the effective magnetic fields acting on each magnetization. Mansuripur has also developed a program using the LLG equation and applied to the wall motion in MO medium[4]. We applied the simulation to the light modulation type overwrite, especially to analysing the magnetization behaviors near the layer boundary in the A-type exchange-coupled bilayer magneto-optical media, amorphous $\mathrm{TbFe} / \mathrm{GdTbFeCo}$, caused by the light strength modulation. The beam fixed model is assumed, in order to reduce computing time.

In the simulation, the LLG equation is rewritten into finite difference equation and the Alternative Direction Implicit (ADI) method is applied[5,6]. The results are shown for the low process and the high process in the light modulation type overewrite. In the following, symbols $R$ and $M$ are used to represent the reference layer and memory layer, respectively.

\section{MEDIA PROPERTIES}

Disk structure and temperature dependence of magnetic parameters for $\mathrm{M}$ and $\mathrm{R}$ are shown in Figs.1 and 2. The Curie points, $\mathrm{T}_{\mathrm{CM}}$ and $\mathrm{T}_{\mathrm{C}, \mathrm{R}}$ are $135^{\circ} \mathrm{C}$ and $325^{\circ} \mathrm{C}$ respectively. The compensation point $T_{\text {Comp, }}$ is $150^{\circ} \mathrm{C}$. $R$ is RE-rich below $T_{\text {COMP R. }}$. $A$ damping parameter, $\alpha$, is 0.3 for $R$ and $M$. We selected the disk shape region with $210 \mathrm{~nm}$ radius as the analysis region and assumed fixed beam irradiating the disk center.

Temperature distribution in radial direction by the pulsed laser beam with $60 \mathrm{~ns}$ duration and $0.1 \mu \mathrm{m}$ beam radius $\left(1 / \mathrm{e}^{2}\right.$ power) Gaussian distribution was calculated by difference method[3]. While, we assumed the uniform temperature in the film thickness direction. Therefore, the magnetic parameters are denoted as functions of the distance from the beam center. So that the cylindrical coordinates system is adopted and the calculation region is described in $(r, z)$ system.

\section{NUMERICAL ANALYSIS SCHEME}

\subsection{Basic equation}

Thic I Stau-Lifhitz-Cilert (LLG) equation , on which our simulation is based on, is denoted by the following form,

$$
\frac{\mathrm{d} \mathbf{M}}{\mathrm{dt}}=-\gamma\left(\mathbf{M} \times \mathbf{H}_{\mathbf{P}}\right)+\frac{\alpha}{M}\left(\mathbf{M} \times \frac{\mathrm{d} \mathbf{M}}{\mathrm{dt}}\right),
$$

where, $M$ is the magnetization vector and $\gamma(>0)$ is gyromagnetic constant. $\mathrm{H}_{\mathrm{p}}$ is the sum of various effective magnetic fields and the explicit expression is derived from the derivative of magnetic energy density. It is important for the simulation to just sufficiently enough take into account effective fields that govern the concerned problem.

\subsection{Effective magnetic fields}

The five kinds of effective magnetic fields are assumed for the nucleation and growth of reverse magnetic domain in the magneto-optical media. We take account of these fields in the following way.

(1)The applied bias field. (2)The demagnetizing field due to the surface magnetic charge of each element. (3)Only the uniaxial anisotropy. (4)The exchange field,which is automatically introduced through exchange interaction between the elements. (5)The coercive field, which is automatically introduced from the spatial fluctuation in magnetic parameters,

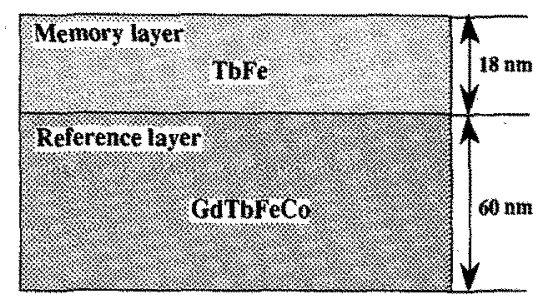

Fig. 1 Bilayer film structure. 


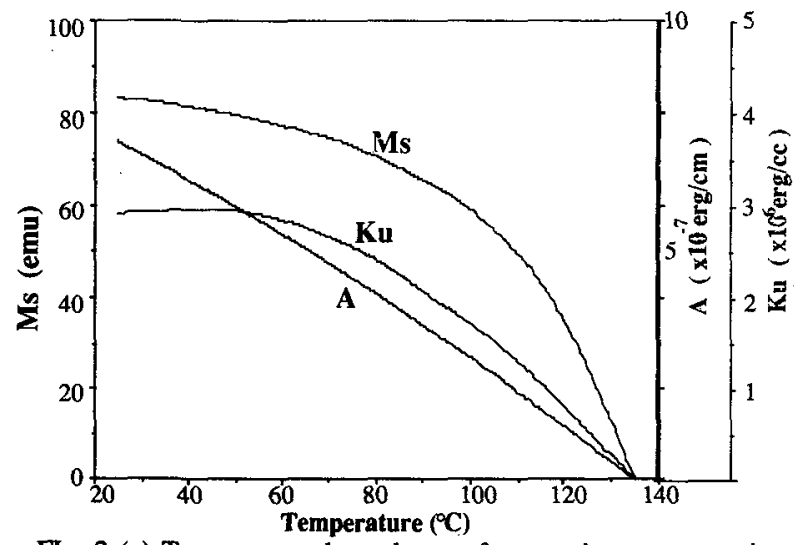

Flg. 2 (a) Temperature dependence of magnetic parameters in memory layer.A, $\mathrm{Ku}$ and $\mathrm{Ms}$ are exchange constant, uniaxial anisotropy constant and magnetization, respectivély.

such as the jump of parameters at bilayer boundary. But we may assume the origin of coercivity when the cause is not clear.

3.3 Finite difference approximation for LLG equation

Magnetization vector is represented in Cartesian coordinates, $\mathbf{M}^{2}=\mathrm{Mx}^{2}+\mathrm{My}^{2}+\mathrm{Mz}^{2}$, since the magnetization is not constrained to specific directions, although the cylindrical coordinates is used for spatial position representation. Explicit expression for $\mathrm{H}_{\mathrm{p}}$ is given as follows;

$$
\begin{aligned}
& \mathrm{E}=\frac{\mathrm{Ku}}{\mathbf{M}^{2}}\left(\mathbf{M}_{\mathrm{x}}^{2}+\mathbf{M}_{\mathrm{y}}^{2}\right)+\frac{\mathrm{A}}{\mathbf{M}^{2}} \sum\left[\left(\frac{\partial \mathbf{M}_{i}}{\partial \mathrm{r}}\right)^{2}+\left(\frac{1}{\mathrm{r}} \partial \mathbf{M}_{\mathrm{i}}\right)^{2}+\left(\frac{\partial \mathrm{M}_{\mathrm{i}}}{\partial \mathrm{z}}\right)^{2}\right] \\
& -\left(\mathbf{H}_{\mathrm{D}}+\mathbf{H}_{\mathrm{B}}\right) \mathbf{M} .
\end{aligned}
$$$$
\mathbf{H}_{\mathbf{P}}=-\operatorname{grad}_{\mathbf{M}} \mathrm{E} \text {, }
$$

Here $A, K u, H_{B}$ and $H_{D}$ are exchange constant, uniaxial anisotropy constant, bias field and the demagnetizing field, respectively. Substituting Eq.(2) into Eq.(1), we obtain the partial differential equation

$$
\frac{\mathrm{d} \mathbf{M}}{\mathrm{dt}}=\mathrm{F}\left(\mathbf{M}, \nabla \mathbf{M}, \nabla^{2} \mathbf{M}\right) \text {. }
$$

In the simulation, Eq.(4) is rewritten in finite difference form and solved as a initial value problem with boundary condition. To guarantee the stability of calculation, we adopted implicit method where the right hand side of Eq.(4) is evaluated at next time, $t+d t$. Finally, we get

$$
\begin{aligned}
\frac{\mathrm{d} \mathbf{M}}{\mathrm{dt}} & =\mathrm{F}\left(\mathbf{M}+\Delta \mathbf{M}, \nabla(\mathbf{M}+\Delta \mathbf{M}), \nabla^{2}(\mathbf{M}+\Delta \mathbf{M})\right) \\
& =\mathrm{F}(\mathbf{M})+\mathrm{F}^{\prime} \cdot \Delta \mathbf{M}+\mathrm{d}\left((\Delta \mathbf{M})^{2}\right) .
\end{aligned}
$$

The time development of each magnetization is given by solving the Eq.(5) with neglecting the term of $O\left((\Delta M)^{2}\right)$. In a two dimensional finite difference equation, the complete implicit method requires very long computing time proportional to $\left(N_{r} \cdot N_{Z}\right)^{2}$ where $N_{r}$ and $N_{Z}$ are the number of elements in the radius and in the film thickness direction, respectively. Therefore, the Alternative Direction Implicit (ADI) method is used to achieve a reduction of computing time to the order of $\mathrm{O}\left(\mathrm{N}_{\mathrm{r}} \cdot \mathrm{N}_{\mathrm{Z}}\right)$, as well as a calculation stability.

Time increment $\mathrm{dt}$ must be chosen sufficiently short, compared with the shortest time scale of a magnetization precession. Spatial increment, $\mathrm{dr}$ and $\mathrm{dz}$, have to be determined to give 4 or 5 calculating points within the magnetic wall width.

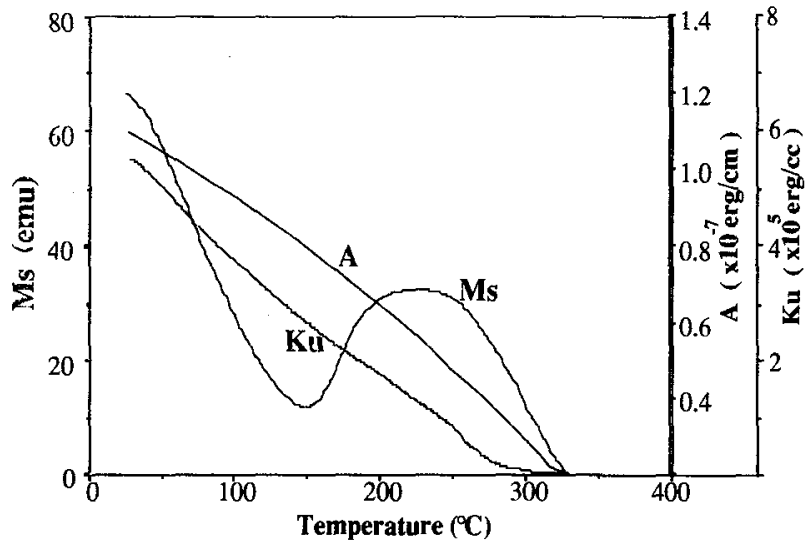

Flg. 2 (b) Temperature dependence of magnetic parameters in reference layer.

That is,

$$
\mathrm{dt} \ll \frac{2 \pi}{\gamma \mathrm{H}_{\mathrm{P}}}
$$

and

$$
\mathrm{dr}, \mathrm{dz}<\pi \Delta_{0} / 4 \text { or } 5
$$

are the necessary conditions for discrete increment size.

3.4 Assumption and corrections

(1) The treatment for $\theta$ derivative of magnetization component Mi;i=x,y,z

When reducing the dimensions for Eqs.(1)-(3) to two-dimension $(r, z)$, we selected either of two kinds of evaluations for $\theta$ derivative of $M$, a uniform phase or radially rotational type, depending on the purpose of the problem.(Fig.3)

(2) Correction for making $M$ constant

In Fo.(5) which is derived as the lincar approximation of basic Eq.(1), the magnitude of magnetization at each point can not be conserved against time development, although that is constant in itself in Eq.(1). So we renormalize the magnitude in the final stage at each time step to cover up the fault in the simulation.

(3) Preparation of newly generated $M$ at $T_{C, M}$ radius

On a cooling process, when the $T_{C}$ radius gradually becomes smaller, it is necessary to give the direction to the newly generated magnetization at $T_{C}$ radius. In the simulation, the newly magnetization direction is prepared in accordance with the $\mathrm{H}_{\mathrm{B}}+\mathrm{H}_{\mathrm{D}}$ direction.

(4) Note on TM magnetization in Ferrimagnet

The vector magnetization $\mathbf{M}$ in LLG equation is represented by the magnitude for net magnetization, Ms and the direction for magnetization of TM( Fe atom) in Ferrimagnet medium, because the dominant exchange interaction in the medium is that of between TM-TM atoms. We use A-type Ferrimagnet here. Then, in $\mathrm{R}$, TM magnetization is naturally antiparallel to net magnetization below $\mathrm{T}_{\text {Comp }}$. In the temperature range below $T_{\text {COMP,R}}$, therefore, we have to carefully assume the demagnetization field and external field directions relative to the net magnetization. Practically, the direction for the de-

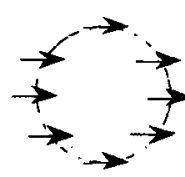

(a)

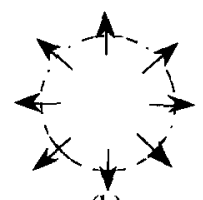

(b)
Fig. 3 (a)The uniform phase type and (b)the radially rotational type. 
magnetizing field generated by the net magnetization and the external field direction were reversed in $\mathrm{R}$.

\section{RESULTS AND DISCUSSIONS}

We have performed two types of simulation, a bit erasure (low-process) and a bit writing (high-process) at $\mathrm{H}_{\mathrm{B}}=500 \mathrm{Oe}$. (a) Low process

The mechanism to erase bit "1" is slown in Fig.4. The domain wall has initially been stabilized in $\mathrm{R}$, near the boundary between $R$ and $M$ by the influence of the initializing field, $\mathrm{H}_{\mathrm{IN}}$. Simulation starts $6 \mathrm{~ns}$ after the ignition of the laser with $1.5 \mathrm{~mW}$. At $6 \mathrm{~ns}$, the temperature was $130^{\circ} \mathrm{C}$ at the irradiation center. The wall positions, defined by the magnetization contour, at which the z-component of magnetization, $\mathrm{Mz}$, is 0 , are consecutively computed, up to $8 \mathrm{~ns}$ under the bias field, $\mathrm{H}_{\mathrm{B}}=5000 \mathrm{O}$. The wall moved upwards and punched through the $R$ surface to $M$, when the temperature rose until higher than $130^{\circ} \mathrm{C}$. After the punch through, the wall velocity drastically decreased, due to the influence of $\mathrm{H}_{\mathrm{B}}$. At 7.3ns, the temperature at the laser irradiation center became higher than $T_{C, M}$, so that the wall extinguished within the $T_{C, M}$ radius. In a norm operation, the peripheral wall edge produced by the extinguishment should continue to move upwards against the $\mathrm{H}_{B}$, until it reached the top surface to $M$. By increasing the exchange constant, $A$, in $M$ to the $\sqrt{10}$ times larger than the above value, the punch through was indeed observed, as shown in Fig.5. The results indicate that the punch through from $R$ and $M$ at near $T_{C, M}$ depends very sensitively on the values of magnetic parameters in $\mathrm{M}$.

As the parameters to dominate the boundary wall motion, we chose the exchange constant, $A_{M}, K_{M}, H_{B}$ and the temperature of medium and carried out some simple simulations for infinitely expanded plane wall model. Fig. 6 shows the dependence of the punch through temperature, $T_{\mathrm{PTH}}$, from $R$ to $M$ on boundary wall width in $M, \delta_{M}$ when $H_{B}=500$ Oe. $T_{P T H}$ is found to increase with decreasing $\delta_{M}$.

In order to understand the above results we consider the simple model which includes the wall energy density, demagnetizing energy and Zeeman energy due to the external field. When the wall center moves to $\mathrm{M}$ by $\mathrm{dz}$, the change of total energy density, $\mathrm{dE}$, is given by

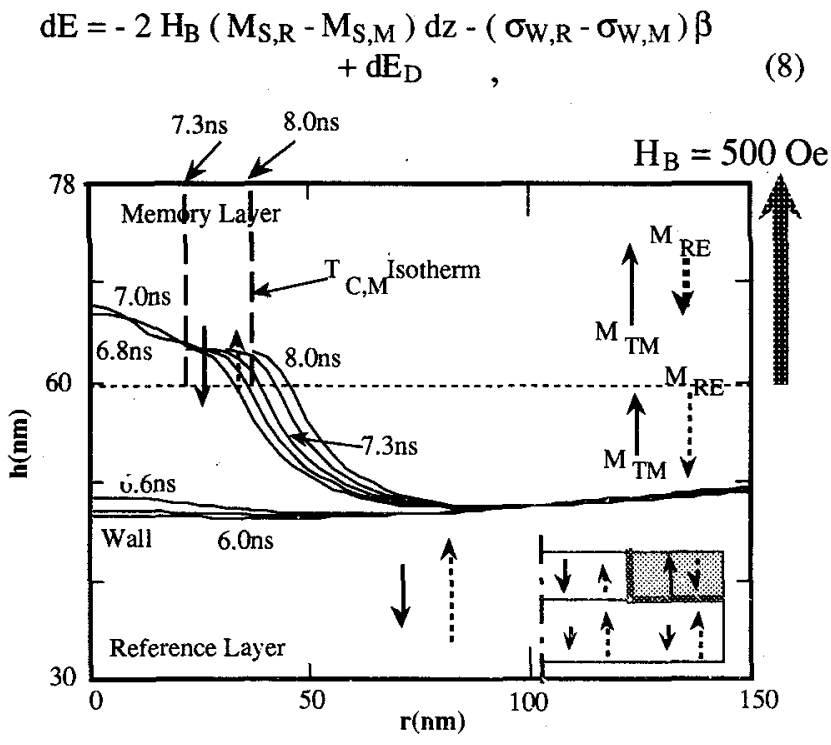

Fig. 4 Low process for erasing bit "1" where the first and second terms in the right hand side are the changes of the Zeeman energy and of wall energy due to penetration of the tail into the M,respectively. $\beta$ is the coefficient proportional to $\mathrm{dz} /\left(\delta_{\mathrm{M}}+\delta_{\mathrm{R}}\right)$. The third term is demagnetizing field energy change induced by the wall movement, but this term is negligible small compared with the other terms in Eq.(8) in our case. At temperature near $T_{C M}$, the first and second terms are negative and the punch through is thought to occur. In the simulation, however, punch through is not always observed even when $\mathrm{dE}<0$. To examine the condition for punch through we take $H_{B}=0$ and get the result that punch through did not realize until temperature reaches to $T_{C, M}$. So these results suggests that we should add a kind of barrier term to Eq.(8). That might be introduced from the change of wall energy near boundary, but it is not simple. The temperature $\mathrm{T}_{\text {PTH }}$ at which the magnetic wall jumps into $\mathrm{M}$, is determined by the condition that the driving force almost equals to this barrier height. Therefore, the fact that $\mathrm{T}_{\mathrm{PTH}}$ shifts to lower temperature with increasing the boundary wall width in $M$, means smaller barrier, because the driving force for a given $H_{B}$ decreases with decreasing temperature.

Once the wall goes into $M$, it punches through easily in the case of larger $\sigma_{\mathrm{M}}$, since $\mathrm{Ms}_{\mathrm{M}}$ is already small at temperature close to $\mathrm{T}_{\mathrm{C}, \mathrm{M}}$.

(b) High process

High process for writing bit " 1 " consists of two stages; magnetization reversal process by $\mathrm{H}_{\mathrm{B}}$ to nucleate a reverse domain in $R$ at near $T_{C, R}$, higher than $T_{C O M P, R}$, and replication of reversed domain from $R$ to $M$ at below $T_{C, M}$. The simulation for the first stage starts at $60 \mathrm{~ns}$ after the laser with $60 \mathrm{~ns}$ pulse duration and $4.0 \mathrm{~mW}$ power has stopped irradiation. Here, $H_{B}$ is 5000 e. Reverse domain grew up from $60.8 \mathrm{~ns}$ to 61 .1ns. After $61.2 \mathrm{~ns}$, however, the domain began to shrink, since the demagnetizing field component, induced by the magnetization in the region, where the temperature is lower than $\mathrm{T}_{\mathrm{COMPR}, \mathrm{R}}$, gradually increased at the domain wall. The reason is that the $T_{C O M P, R} r a-$ dius shrinks with decreasing the temperature, as shown in Fig.7. Therefore $T_{C, M}$ radius ( $>T_{\text {comP } R}$ radius) never catches up and passes the reverse domain wall in the $R$.

Then, in order to realize the high process simulation, we trapped the domain wall at a radius less than the maximum domain radius at $61.1 \mathrm{~ns}$, by setting the artificial $\mathrm{H}_{\mathrm{C}}$. A practical $\mathrm{H}_{\mathrm{C}}$ is given by making a sinusoidal modulation for the uniaxial

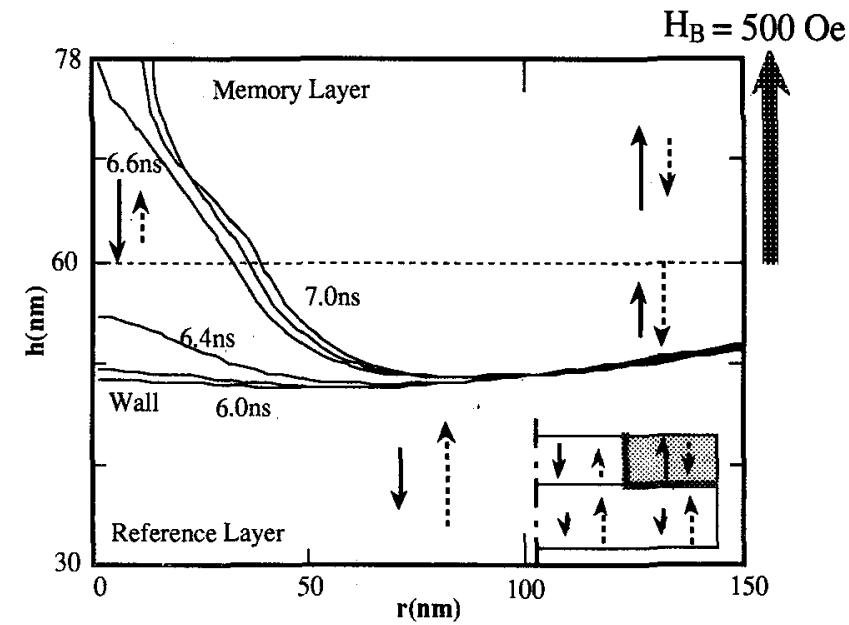

Fig. 5 The same process as Fig. 4 with the parameter $A_{M}$ as $\sqrt{10}$ times larger than in Fig. 4. 


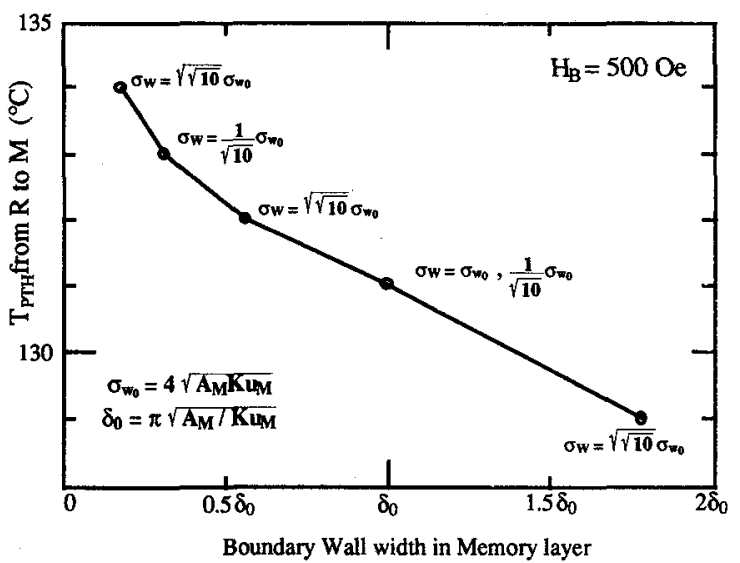

Fig. 6 The punch through temperature from $R$ to $M$ vs. the boundary wall width in $\mathrm{M}, \boldsymbol{\delta}_{\mathrm{M}}$

anisotropy energy with a periods of $2 \pi \delta_{R}$ in the radial direction over the thickness in $R$. The second simulation started at $66 \mathrm{~ns}$. When the $T_{C, M}$ radius reached nearly one half of the calculation region radius, $210 \mathrm{~nm}$. The results are shown in Fig. $8 . \mathrm{T}_{\mathrm{C}, \mathrm{M}}$ isotherm moved towards left as time passed and about $66.8 \mathrm{~ns}$, the isotherm traversed the top of the domain wall in $R$. At $67.2 \mathrm{~ns}$, the domain wall in $\mathbf{R}$ started to move towards left overcoming artificial $H_{C}$ in the vicinity of the layer boundary and after $67.4 \mathrm{~ns}$, the whole magnetic domain wall began to move. The temperature of the bit center dropped below $T_{C . M}$ around $67.7 \mathrm{~ns}$. Then the replication from $R$ to $M$ was proved in the region near the irradiation center.

We discuss the results by writing process in each single layer forming the medium to understand the properties of exchange-coupled bilayer medium. Our simulation was done as follows: after heating to above $T_{C}$, writing was performed in $H_{B}=5000$ e for each layer. Writing was failed in $M$. The failure in $M$ is due to large $H_{K}$ making nucleation difficult and the wall shrinking forces making the reversed domain unstable. In $R$, we could get reverse magnetic domain in the first few $n s$, already shown Fig.7.

Eventually, we have successfully written in bilayer, which is a composite film of the above mentioned $R$ and $M$, at $H_{B}=$ 5000 e. This result means that the exchange-interaction between the two layers creates very large effective magnetic field for replicating a reverse domain into $\mathrm{M}$.

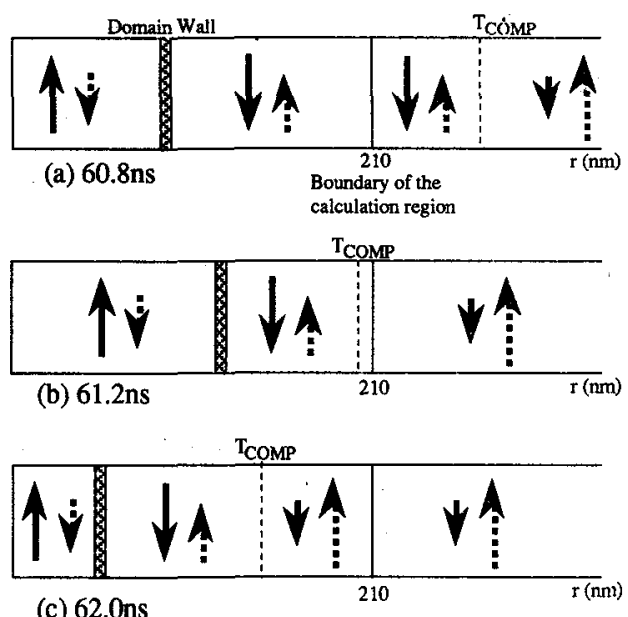

(c) $62.0 \mathrm{~ns}$

Fig. 7 Wall position dependence on $T_{\text {m }}$ radius in $R$ for high process. The whole magnetization of outer region are considered same as that at boundary in the simulation. Therefore the $T_{\text {comp, }}$ radius gives the pressure on the wall, when it traverses the calculation boundary from right to left as shown (a),(b) and (c).

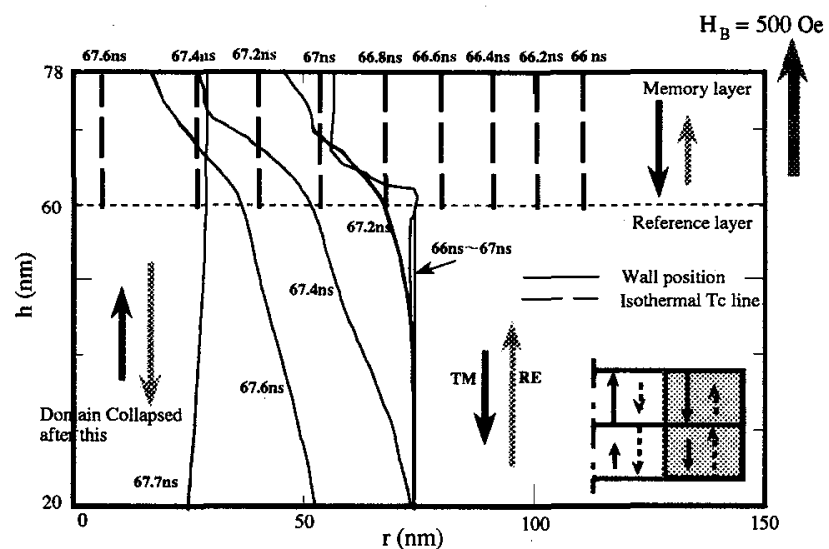

Fig. 8 Replication process of reverse magnetic domain form $R$ to $M$

The written bit "1" must be remained stable after initialization process for $\mathrm{R}$ by $\mathrm{H}_{\mathrm{IN}}$ at room temperature, in other word the boundary magnetic wall have to stay in $R$ (in our case) even when $\mathrm{H}_{\mathrm{B}}=0$. It is confirmed that this condition is satisfied with parameters in this simulation. However, Kaneko et al, reported that the boundary wall punched through from the layer with smaller $\sigma_{w}$ at $H_{B}=0$ in bilayer system[7]. Whether the boundary wall stays in film stable or punches through from the film at $H_{B}=0$ seems to depend on the relation between the wall width and the film thickness.

\section{CONCLUSION}

The computer simulation based on Landau-Lifshitz-Gilbert equation was developed and applied to analysing the reverse magnetic domain generation or its erasure in the light modulation overwrite medium, the exchange-coupled amorphous $\mathrm{TbFe} / \mathrm{GdTbFeCo}$ bilayer film. It was found that the exchange coupling between the reference and the memory layers plays an essential role in replicating the domain from the reference to the memory layer. The dynamics of the boundary wall, which has a important role in the erasure, was discussed, based on the correlation between the boundary wall width in $M, H_{B}$ and the temperature.

\section{ACKNOWLEDGMENTS}

The authors would like to thank Dr.S.Esho and Dr.T.Kumamoto for their support and encouragement and Dr.H.Gokan for his support and stimulating discussions on the simulation. Thanks are also due to Messrs.T.Habara, K.Hayashi and S.Shimizu for the heat flow calculation and property characterization.

\section{REFERENCES}

[1] T. Nakano, et al: Jpn. Appl. Phys. , Suppl. , 26-4,149(1987) [2] J. Saito, et al: Proc. Int. Symp. on Optical Memory, Tokyo 1987, Jpn. Appl. Phys. , Suppl. , 26-4, 155(1987)

[3] M. Okada, et al: The 13th Annual Conf. Magn. Jpn. , 22pC9(1989)

[4] M: Mansuripur : J. Appl. Phys. , 63, 5809(1988)

[5] M. Hasegawa, et al: Papers of Tech. Meeting on Magn. , MAG-91-26, IEE Jpn.(in Japanese)

[6] M. Hasegawa, et al: IEEE Trans. Magn. , MAG-22, 802(1986)

[7] M. Kaneko, et al: Jpn. Phys. , Suppl. , 28-3, 27(1989) 